\title{
Rush immunotherapy with multiple aeroallergens is safe in an adult population*
}

\author{
Wytske Fokkens'1, Maurits van Maaren², Marije Wolvers', Roy Gerth van \\ Wijk $^{2}$
}

'Department of Otorhinolaryngology, Academic Medical Centre, Amsterdam, the Netherlands 2Section of Allergology, Department of Internal Medicine, Erasmus Medical Center, Rotterdam, the Netherlands
Rhinology Online, Volume 1: 35 - 37, 2018

http://doi.org/10.4193/RHINOL/18.019

*Received for publication:

April 20, 2018

Accepted: April 26, 2018

Published: May 1, 2018

\begin{abstract}
Background: The safety of rush subcutaneous immunotherapy (SCIT) to multiple aeroallergens has not been widely studied. The objectives of this retrospective study were to evaluate the incidence of adverse reactions of a rush immunotherapy scheme with five injections at the first day with different allergens in adult patients with allergic rhinitis with or without asthma. All patients took an antihistamine before the start of the first injection of the rush scheme.
\end{abstract}

Methods: adverse reactions were analysed in a prospectively recorded database of 232 rush SCIT treatments with tree pollen, given to 138 patients with allergic rhinoconjunctivitis (61 (44\%) male), mean age 31 years old (9-57), 45 (33\%) with concomitant asthma, and 28 (20\%) with eczema.

Results: Systemic adverse reactions (gr 1-2) were reported 7 times (3\%), one patient on house dust mite (HDM) complained of shortness of breath and had a reduction in peak expiratory flow (PEF) of 10\% (gr 2). The other 6 patients ( 4 on HDM and 2 on GP) complained of mild upper respiratory symptoms mostly itchy throat. Four of the 7 participants who had systemic adverse reactions, had also received at least one rush SCIT with another allergen in the week before. A local reaction (with redness and swelling) was reported in 13 patients. Two HDM SCIT rushes were aborted, and patients proceeded on a conventional schedule.

Conclusion: Rush SCIT with 5 injections on the first day of the treatment does not give more adverse reactions than what is usually reported with conventional immunotherapy.

Key words: immunotherapy, rush immunotherapy, allergic rhinitis, allergic rhinoxonjunctivitis, treatment, adverse reactions, systemic, local reaction

\section{Introduction}

Subcutaneous allergen immunotherapy (SCIT) is effective in improving symptom, medication, and combined symptom and medication scores in patients with allergic rhinoconjunctivitis while on treatment, and there is some evidence suggesting that these benefits are maintained in relation to symptom scores after discontinuation of therapy ${ }^{(1)}$. SCIT protocols are performed in two stages: build-up (up-dosing) phase which involves the administration of increasing doses of allergen extracts until the effective (or maintenance) dose is reached, and the maintenance phase. Conventional immunotherapy schedules generally involve one or two weekly injections during up-dosing phase, over a 16-week period, followed by monthly maintenance injections for a period of three to five years. Rush and cluster immunotherapy schedules are accelerated build-up schedules which allow the patient to reach the maintenance dose and, thus, the benefits of immunotherapy, more rapidly. Several cluster (consisting of 2-4 repeated injections given to the patient in a single day of treatment on nonconsecutive days, in most cases reaching the maintenance dose in four to eight weeks) and rush schedules (consisting of repeated injections with increasing amounts of allergen extracts at intervals of 15-60 min over a period ranging from one to three days at the beginning of an immunotherapy build-up dosing have been described ${ }^{(2)}$. Acce- 
Table 1. Reactions to rush SCIT.

\begin{tabular}{|c|c|c|c|c|c|c|c|c|}
\hline \multirow[b]{2}{*}{ Reaction } & \multicolumn{2}{|c|}{$\begin{array}{c}\text { Tree } \\
(\mathbf{N}=\mathbf{7 5})\end{array}$} & \multicolumn{2}{|c|}{$\begin{array}{l}\text { Grass } \\
(\mathbf{N}=\mathbf{8 9})\end{array}$} & \multicolumn{2}{|c|}{$\begin{array}{l}\text { HDM } \\
(N=66)\end{array}$} & \multicolumn{2}{|c|}{$\begin{array}{c}\text { Total } \\
(\mathrm{N}=\mathbf{2 3 0})\end{array}$} \\
\hline & $\mathrm{N}$ & $\%$ & $\mathrm{~N}$ & $\%$ & $\mathrm{~N}$ & $\%$ & $\mathrm{~N}$ & $\%$ \\
\hline Systemic reaction (gr 1) & 0 & $0 \%$ & 2 & $2 \%$ & 4 & $6 \%$ & 6 & $3 \%$ \\
\hline Systemic reaction (gr 2) & 0 & $0 \%$ & 0 & $0 \%$ & 1 & $2 \%$ & 1 & $0.4 \%$ \\
\hline Local reaction & 3 & $4 \%$ & 9 & $10 \%$ & 1 & $2 \%$ & 13 & $6 \%$ \\
\hline $\begin{array}{l}\text { Systemic reaction not linked to immunotherapy } \\
\text { (according to 7) }\end{array}$ & 2 & $3 \%$ & 5 & $6 \%$ & 4 & $6 \%$ & 11 & $5 \%$ \\
\hline Limited local redness/itch without swelling & 2 & $3 \%$ & 3 & $3 \%$ & 3 & $5 \%$ & 8 & $3 \%$ \\
\hline
\end{tabular}

lerated subcutaneous immunotherapy (SCIT) schedules provide significant (time) benefits over conventional immunotherapy for the patients but has potentially more serious adverse reactions (3). The safety of rush immunotherapy to multiple aeroallergens has not been widely studied ${ }^{(4-6)}$. We frequently use a rush immunotherapy scheme with Alutard SQ ${ }^{\circledR}$ (ALK-Albello) SCIT involving the first 5 injections out of a total of 15 injections which makes up the up-dosing phase on the first day, followed by weekly injections. The objectives of this retrospective study were to evaluate the incidence of adverse reactions of this rush immunotherapy scheme used in daily practice with different allergens in adult patients with allergic rhinitis with or without asthma.

\section{Materials and methods}

A retrospective review of 138 patients with allergic rhinoconjunctivitis receiving rush SCIT with multiple aeroallergens in a university-based allergy practice was conducted. All patients received premedication with oral non-sedating antihistamines When rush SCIT was given for more than one allergen the time in between the start of the next rush SCIT was at least 3 days, but mostly 1 week. Patients always received one allergen only at the day of the rush SCIT.

Immunotherapy was carried out with tree pollen (Alutard SQ 197), grass pollen (Alutard SQ 293), HDM (Alutard SQ 503 ) and cat (Alutard SQ555) from ALK-Abello, Hørsholm, Denmark

Data of the patients like age, gender, prevalence of asthma and/ or eczema and adverse reactions were recorded prospectively in a database. Side effects were noted as they were reported by the patients and later categorized according to World Allergy Organization Subcutaneous Immunotherapy Systemic Reaction Grading System ${ }^{(7)}$.

An analysis of the adverse reactions in relation to allergen, number of allergens used and period in between the rush SCIT was made.

\section{Results}

138 patients with allergic rhinoconjunctivitis (61 (44\%) male), mean age 31 years old (9-57), 45 (33\%) with concomitant asthma, and 28 (20\%) with eczema were given a total of 232 rush SCIT treatments with one or more allergens between September 2014 and January 2018: 75 for tree pollen , 89 for grass pollen, 66 for HDM, and 2 for cat. A significant percentage of the patients received SCIT with more than one allergen: 19 patients received birch, grass and HDM, 31 received birch and grass together, and 13 received grass and HDM, one patients received birch and cat.

The frequencies of the side effect for birch, grass, and HDM rush SCIT are shown in Table 1.

Systemic adverse reactions (gr 1-2) ${ }^{(7)}$ were reported 7 times, one patient on HDM complained of shortness of breath and had a reduction in PEF of $10 \%$. He was treated with salbutamol inhalation. The other 6 patients ( 4 on HDM and 2 on GP) complained of mild upper respiratory symptoms mostly itchy throat. Of the 7 patients ( 5 asthmatics) who had systemic adverse reactions, 4 had also received at least one rush SCIT for another allergen in the week before.

A local reaction (with redness and swelling) was reported in 13 patients (7 asthmatics). Most of these patients did not receive a rush SCIT for another allergen in the two weeks before. One patients had a local reaction at rush for tree pollen and GP but not HDM. Eleven patients complained about systemic effects which are usually not reported as a part of an acute allergic reaction to be related to immunotherapy like tiredness ${ }^{(7)}$. Eight patients had a limited local redness without swelling.

Two SCIT rushes were discontinued, and proceeded on a conventional schedule. Both were treated with HDM. The first rush was abrogated because of itch of the throat and ears. This participant also had a tree pollen rush 11 days before, and a grass rush 3 days before. The second was abrogated because of "feeling cold and tired and itch". This participant did not have a rush SCIT prior to the HDM rush. Both participants were not asthmatic and had no eczema. In both cases medication for these symptoms was not required. 


\section{Discussion}

This evaluation showed that rush treatment with 5 injections on the first day followed by weekly injections does not result in more adverse reactions than conventional immunotherapy. The number of adverse events to tree pollen immunotherapy was less than seen in immunotherapy with grass pollen and HDM. An explanation could be that tree pollen rush treatment - regularly given first because of the order of seasons- leads to adverse events during following rush treatments. An alternative explanation is that the nature of the allergens determines the number of side effects.

The safety profile of allergen immunotherapy (AIT) has been thoroughly documented in well-designed clinical studies ${ }^{(8-10)}$. Also, recently, the Immunotherapy Interest Group of the European Academy of Allergy and Clinical Immunology (EAACI) reported, based on a prospective, longitudinal, web-based survey of 'real-life' respiratory allergen immunotherapy (AIT), that AIT for respiratory allergy is safe, with a low number of adverse reactions observed in real-life clinical practice ${ }^{(11)}$. In a total of 3398 SCIT treatments 97 (3\%) systemic adverse reactions were reported. The most frequently reported symptoms were urticaria, rhinitis, dyspnoea and cough.

Most systemic adverse reactions occurred during the up-dosing phase (75.8\%) and were mild in severity (71.6\%). Independent risk factors for systemic adverse reactions were: the use of natural extracts, the absence of symptomatic allergy medications, a diagnosis of asthma, sensitization to animal dander or pollen and cluster regimens (vs rush). In this study, we found the same as reported in the real life clinical practice study: $3 \%$ systemic adverse reactions, usually mild and in the upper airways. The number of adverse reactions was not large enough to further analyse risk factors but it is an interesting observation that half of the systemic adverse reactions occurred in patients that started a next rush SCIT for a different allergen within a week of the first rush SCIT treatment. This is a conclusion that is not illogical but that could not be confirmed by data published in the literature.

\section{Conclusion}

Rush SCIT with 5 injections on the first day of the treatment does not give more adverse reactions than what is usually reported with conventional immunotherapy in this group of 232 rush SCIT with BP, GP and HDM.

\section{Acknowledgements}

We thank Wilma van Bergen Henegouwen voor putting together the database.

\section{Authorship contribution}

WF wrote the study protocol, analysed data and wrote the manuscript. MvM collected the data, corrected and approved the manuscript. MW analysed data, corrected and approved the manuscript. RGvW collected data, corrected and approved the manuscript.

\section{Availability of data and materials}

The datasets generated and/or analysed during the current study are available in the Figshare repository, 10.6084/ m9.figshare.7338953.

\section{Conflict of interest}

None
1. Dhami S, Nurmatov U, Arasi S, Khan T, Asaria $M$, Zaman $\mathrm{H}$, et al. Allergen immunother apy for allergic rhinoconjunctivitis: A systematic review and meta-analysis. Allergy. 2017;72(11):1597-631

2. Alvarez-Cuesta E, Bousquet J, Canonica GW, Durham SR, Malling HJ, Valovirta E. Standards for practical allergen-specific immunotherapy. Allergy. 2006;61 Suppl 82:1-20.

3. Gomes Dos Reis Pimentel RA, Oliveira G, Ferreira Chaves Loureiro ELCS. Accelerated subcutaneous immunotherapy in pediatric population - Systematic review. Pulmonology. 2017 Dec 1. pii: S2173 5115(17)30152-5.

4. Brehler R, Klimek L, Pfaar O, Hauswald B, Worm M, Bieber T. Safety of a rush immunotherapy build-up schedule with depigmented polymerized allergen extracts. Allergy Asthma Proc. 2010 MayJun;31(3):e31-8.

5. Buczylko K, van der Werf JF, Boot D, van Ree R. Accelerated Up-Dosing of Subcutaneous
Immunotherapy with a Registered Allergoid Birch Pollen Preparation. Int Arch Allergy Immunol. 2017;172(3):183-186.

6. Pfaar O, Urry Z, Robinson DS, Sager A, Richards D, Hawrylowicz CM, et al. A randomized placebo-controlled trial of rush preseasonal depigmented polymerized grass pollen immunotherapy. Allergy. 2012;67(2):272-9.

7. Cox L, Larenas-Linnemann D, Lockey RF Passalacqua G. Speaking the same Ianguage: The World Allergy Organization Subcutaneous Immunotherapy Systemic Reaction Grading System. J Allergy Clin Immunol. 2010;125(3):569-74, 74.e1-74.e7.

8. Ceuppens $\mathrm{JL}$, Bullens D, Kleinjans $\mathrm{H}$, van der Werf J. Immunotherapy with a modified birch pollen extract in allergic rhinoconjunctivitis: clinical and immunological effects. Clin Exp Allergy. 2009 Dec;39(12):1903-9

9. Pfaar O, Nell MJ, Boot JD, Versteeg SA, van Ree $\mathrm{R}$, Roger A, et al. A randomized, 5-arm dose finding study with a mite allergoid
SCIT in allergic rhinoconjunctivitis patients. Allergy. 2016;71(7):967-76

10. Walker SM, Varney VA, Gaga M, Jacobson MR, Durham SR. Grass pollen immunotherapy: efficacy and safety during a 4-year follow-up study. Allergy. 1995;50(5):405-13.

11. Calderon MA, Vidal C, Rodriguez Del Rio P, Just J, Pfaar O, Tabar Al, et al. European Survey on Adverse Systemic Reactions in Allergen Immunotherapy (EASSI): a real-life clinical assessment. Allergy. 2017;72(3):46272.

Wytske Fokkens

Department of Otorhinolaryngology

Academic Medical Centre

1105AZ Amsterdam

The Netherlands

E-mail: w.j.fokkens@amc.nl 